\title{
Author reply: Neoadjuvant targeted therapy in renal cell carcinoma
}

\section{Vincenzo Ficarra and Giacomo Novara}

The results obtained following use of new drugs that target angiogenesis and/or the mammalian target of rapamycin (mTOR) pathway stimulated new interest from oncologists in the medical treatment of metastatic and locally-advanced renal cell carcinoma (RCC). The poor results reported for immunotherapy and chemoimmunotherapy protocols heightened enthusiasm for neoadjuvant and adjuvant medical treatments in the era of 'targeted therapy'. To date, there are no data from randomized controlled trials (RCTs) to support the use of either neoadjuvant or adjuvant targeted therapies in patients with metastatic RCC or nonmetastatic high-risk locally-advanced disease. As stated in our News \& Views article (Kidney cancer: neoadjuvant targeted therapies in renal cell carcinoma. Nat. Rev. Urol. 7, 63-64; 2010), ${ }^{1}$ the use of targeted therapies before surgical resection of primary, recurrent or metastatic RCC should be considered investigational, and be restricted to patients enrolled in RCTs. Careful selection of patients for neoadjuvant therapy is essential if the potential benefits of this multimodal approach are to be appropriately evaluated and the risk of perioperative complications minimized.

In their correspondence (Neoadjuvant targeted therapy in renal cell carcinoma. Nat. Rev. Urol. 7, doi:10.1038/ nrurol.2010.2-c1; 2010), ${ }^{2}$ Iacovelli et al. outline another potential use of neoadjuvant medical therapy in patients with nonmetastatic high-risk disease identified using an integrated prognostic system (that is, the UCLA Integrated Staging System [UISS]), rather than using the TNM classification. We believe this proposition is based on a conceptual mistake and lacks an evidence-based rationale.

Firstly, the UISS cannot be applied to the presurgical decision-making phase because such an integrated system requires that pathological extent of primary tumor (pT) and Fuhrman nuclear grade be defined using nephrectomy specimens. ${ }^{3}$ In 2009, Karakiewicz et al. proposed a nomogram for prediction of cancer-specific survival probabilities based only on preoperative clinical features; the prognostic accuracies were $86.8 \%$ and $84.2 \%$ at 5 years and 10 years, respectively. ${ }^{4}$ We believe that this clinical variable-based nomogram might be a better tool with which to stratify preoperatively nonmetastatic patients, and to identify those at increased risk who are potentially suitable for enrollment in RCTs designed to evaluate the efficacy of neoadjuvant therapies.

Secondly, the lack of data supporting adjuvant treatments in nonmetastatic high-risk patients is not valid motivation for the use of another non-evidence-based strategy such as neoadjuvant therapy. To date, there are no clinical data which indicate that the neoadjuvant approach results in better disease control, regardless of patient risk stratification.

Finally, another potential advantage of neoadjuvant therapy is downsizing of unresectable primary tumors. As stated in our article, ${ }^{1}$ it is unlikely that the limited decrease in mean tumor size reported in the published literature would have a meaningful impact on conversion of initially unresectable tumors to resectable lesions, at least in the hands of experienced urologic surgeons.

In conclusion, the increasing interest in medical therapy for RCC must be correctly oriented, on the basis of evidence, rather than on opinion. Urologists and oncologists must share their knowledge in order to plan well-designed RCTs that can provide answers to (re)opened questions in the era of targeted therapies. We await the results of ongoing RCTs, which might clarify some of the issues regarding the role of targeted therapies in the adjuvant and neoadjuvant settings.

Department of Oncological and Surgical Sciences, Urology Clinic, University of Padua, Monoblocco Ospedaliero-IV Floor, Via Giustiniani 2, 35128 Padua, Italy (V. Ficarra, G. Novara).

Correspondence to: V. Ficarra vincenzo.ficarra@unipd.it

\section{doi:10.1038/nrurol.2010.2-c2}

Competing interests

The authors declare no competing interests.

1. Ficarra, V. \& Novara, G. Kidney cancer: neoadjuvant targeted therapies in renal cell carcinoma. Nat. Rev. Urol. 7, 63-64 (2010).

2. lacovelli, R., Raimondi, C., Palazzo, A., Cortesi, E. \& Procopio, G. Neoadjuvant targeted therapy in renal cell carcinoma. Nat. Rev. Urol. 7, doi:10.1038/nrurol.2010.2-c1.

3. Ficarra, V., Galfano, A., Mancini, M., Martignoni, G. \& Artibani, W. TNM staging system for renal-cell carcinoma: current status and future perspectives. Lancet Oncol. 8, 554-558 (2007).

4. Karakiewicz, P. I. et al. A preoperative prognostic model for patients treated with nephrectomy for renal cell carcinoma. Eur. Urol. 55, 452-459 (2009). 